\title{
Von Überschwemmungen und anderen Ereignissen
}

\author{
Andreas Klee ${ }^{1}$ \\ Online publiziert: 15. Juni 2018 \\ ๑) Springer-Verlag GmbH Deutschland, ein Teil von Springer Nature 2018
}

In den letzten Jahren ist Deutschland immer wieder von hochwasserbedingten Extremereignissen heimgesucht worden. Die Überschwemmungen an Elbe, Rhein, Donau und weiteren Flüssen sind uns noch immer gegenwärtig. Unmittelbar nach schweren Niederschlägen und Hochwassern wird stets der Ruf nach besseren vorbeugenden Schutzmaßnahmen laut. Auch in der räumlichen Planung, allen voran in der Regional- und Bauleitplanung, sowie in den betreffenden Fachplanungen, werden verstärkt innovative Maßnahmen im Rahmen des sogenannten Hochwasserrisikomanagements diskutiert. Zumeist geht es darum, überschwemmungsgefährdete Gebiete von künftiger Bebauung frei zu halten. Dass dies keinesfalls selbstverständlich ist, zeigen zum Teil heftige Diskussionen in den betroffenen Gemeinden, wenn die Belange des Hochwasserschutzes im Rahmen der Abwägung (plötzlich) gegenüber vermeintlich notwendigen Bauprojekten nicht mehr so wichtig sind. Stefan Greiving und Kollegen nehmen dieses immer wieder auftretende Dilemma zum Anlass, über eine andere, radikaler anmutende Strategie nachzudenken. In ihrem Beitrag „Siedlungsrückzug als planerische Strategie zur Reduzierung von Hochwasserrisiken“ skizzieren sie Möglichkeiten eines proaktiven und reaktiven Siedlungsrückzugs, um Überschwemmungen wieder mehr Raum zu geben. Als Beispiel haben die Autoren die Option eines proaktiven Siedlungsrückzugs im Kontext des Stadtumbaus ausgewählt. Wie diese Strategie aussehen kann, wird anhand eines Planspiels in Sachsen gezeigt.

Allen gängigen Prognosen zufolge werden wir künftig mit mehr Überschwemmungen aufgrund zunehmender Extremwetterereignisse im Rahmen des Klimawandels rechnen müssen. In der Diskussion um die Ursachen des Klimawandels ist unstrittig, dass seine Auswirkungen nicht nur aus den klimatischen Veränderungen selbst resultieren, son-

Prof. Dr. Andreas Klee

klee@arl-net.de

1 Akademie für Raumforschung und Landesplanung, Leibniz-Forum für Raumwissenschaften, Hohenzollernstraße 11, 30161 Hannover, Deutschland dern dass auch gesellschaftliche Entwicklungsprozesse und menschliches Handeln eine bedeutende Rolle spielen. Was liegt näher, als die beiden gegenwärtigen „Megatrends“ demographischer Wandel und Klimawandel - zusammen zu denken? Viola Schulze Dieckhoff, Dennis Becker, Stefan Greiving und Thorsten Wiechmann haben dies getan. In ihrem Beitrag „Raummuster: Demographischer Wandel und Klimawandel in deutschen Städten" betrachten sie Klimasignale und demographische Indikatoren gemeinsam und leiten daraus eine Reihe von räumlichen Mustern ab. So zeigen sie beispielsweise, dass in wärmeren Klimaraumtypen die städtische Bevölkerung besonders zunimmt. Dies schafft für die betreffenden Kommunen neue Herausforderungen, insbesondere bei gleichzeitiger Zunahme des Anteils älterer, klimasensiblerer Menschen. Sollte vor diesem Hintergrund die planerische Leitvorstellung der Innenentwicklung überdacht werden?

In einem weiteren Beitrag dieser Ausgabe von „Raumforschung und Raumordnung I Spatial Research and Planning" untersuchen Patrick Küpper und Tobias Mettenberger berufliche und private Standortfaktoren für die Niederlassung von Hausärzten in ländlichen Räumen. Sie greifen damit ein aktuelles Thema auf, das vor allem in ländlichperipheren Regionen für große Schlagzeilen sorgt - Stichwort „Ärztemangel“. Die Ergebnisse ihres Beitrags zeigen jedoch, dass es gar nicht so sehr die „,weichen“ Standortfaktoren wie ein hochwertiges kulturelles Angebot oder vielfältige Freizeitmöglichkeiten sind, die über die Wahl eines Praxisstandortes entscheiden, sondern eher berufliche Aspekte wie die ökonomische Situation der Praxis oder ein enger Patientenkontakt, sowie biographische Bezüge in die Regionen.

Schließlich widmen sich Claus-Christian Wiegandt und Kollegen einem Thema, das mittlerweile Einzug in die Lebenswirklichkeit der meisten Menschen in Deutschland gefunden hat: dem Online-Handel. In ihrem Beitrag „Determinanten des Online-Einkaufs - eine empirische Studie in sechs nordrhein-westfälischen Stadtregionen" untersuchen sie, wer aus welchen Gründen welche Produkte über das Internet erwirbt. Aus räumlicher Sicht ist durchaus überraschend, dass die Entscheidung für den Online-Einkauf 
nur in einem geringen Zusammenhang mit der Attraktivität von Innenstädten und sonstigen Einkaufsorten steht. Ausschlaggebender sind demographische und sozioökonomische Faktoren. So spielen das Geschlecht, das Alter und die Lebensstile eine wesentliche Rolle. Diese Befunde haben erhebliche Konsequenzen für die Positionierung inner- städtischer Einzelhandelsbereiche sowie für die Stadtplanung, die mehr als bisher unterschiedliche Zielgruppen in den Fokus nehmen muss.

Allen Leserinnen und Lesern wünschen wir eine anregende Lektüre. 